\title{
Assessing the impact of climate change on vector-borne viruses in the EU through the elicitation of expert opinion
}

\author{
P. GALE ${ }^{1 *}$, A. BROUWER ${ }^{1}$, V. RAMNIAL ${ }^{1}$, L. KELLY ${ }^{1}$, R. KOSMIDER ${ }^{1}$, \\ A. R. FOOKS ${ }^{2}$ AND E. L. SNAR Y ${ }^{1}$ \\ ${ }^{1}$ Centre for Epidemiology and Risk Analysis, Veterinary Laboratories Agency, Weybridge, Addlestone, Surrey, \\ $U K$ \\ ${ }^{2}$ Rabies and Wildlife Zoonoses Group, Veterinary Laboratories Agency, Weybridge, Addlestone, Surrey, UK
}

\section{SUMMARY}

Expert opinion was elicited to undertake a qualitative risk assessment to estimate the current and future risks to the European Union (EU) from five vector-borne viruses listed by the World Organization for Animal Health. It was predicted that climate change will increase the risk of incursions of African horse sickness virus (AHSV), Crimean-Congo haemorrhagic fever virus (CCHFV) and Rift Valley fever virus (RVFV) into the EU from other parts of the world, with African swine fever virus (ASFV) and West Nile virus (WNV) being less affected. Currently the predicted risks of incursion were lowest for RVFV and highest for ASFV. Risks of incursion were considered for six routes of entry (namely vectors, livestock, meat products, wildlife, pets and people). Climate change was predicted to increase the risk of incursion from entry of vectors for all five viruses to some degree, the strongest effects being predicted for AHSV, CCHFV and WNV. This work will facilitate identification of appropriate risk management options in relation to adaptations to climate change.

Key words: Animal pathogens, arboviruses, climate - impact of, risk assessment.

\section{INTRODUCTION}

The introduction and spread of livestock viruses into the European Union (EU) has substantial veterinary and economic consequences and, in some cases, could impact on human health. Many viruses which infect livestock and humans are transmitted by arthropod vectors such as biting midges, mosquitoes, ticks and sand-flies. Vector-borne livestock viruses, which infect humans include Crimean-Congo haemorrhagic fever virus (CCHFV), Rift Valley fever virus (RVFV)

\footnotetext{
* Author for correspondence: Dr P. Gale, Centre for Epi demiology and Risk Analysis, Veterinary Laboratories Agency, Woodham Lane, Addlestone, Surrey KT15 3NB, UK. (Email: p.gale@vla.defra.gsi.gov.uk)
}

and West Nile virus (WNV). CCHFV, for example, is endemic in parts of eastern Europe and may be transmitted from human-to-human nosocomially and from infected livestock meat to humans [1], with higher incidence in abattoir workers and butchers in Iran [2]. Climate change will impact not only on the distribution and abundance of vectors but also on the interaction between the virus and its vector [3, 4]. Recently, for example, bluetongue virus (BTV) serotype 8 has emerged in north-western Europe including the UK as a result of higher temperatures facilitating transmission of the virus by indigenous midge vectors of the Culicoides obsoletus complex [5, 6]. Since 1998, incursions of BTV in southern Europe have occurred due to the northwards expansion in range of the 
traditional vector, Culicoides imicola [7]. The recent emergence of BTV in livestock in northern Europe highlights the need to understand the potential effects of climate change on the occurrence, distribution and prevalence of livestock diseases. Although the potential problems associated with climate change are now becoming widely recognized, quantitative data on the distribution and abundance of many vector species within the EU are not available and the influence of climate change on the distribution of the vectors is highly uncertain. In addition to the vector route, there are other routes of transmission for viruses to livestock in EU member states, including international trade in livestock, importation of meat products and companion animals, and exposure to wildlife. Transmission of viruses through some routes will be more susceptible to the effects of climate change than other routes.

It is important to consider climate change in combination with other factors [4, 8]. Host ecology, host behaviour and increased globalization including the transportation both of people and cargo containers are important [9]. Recently, transmission of chikungunya virus (CHIKV) was reported for the first time outside the tropics with the emergence of cases in southern Europe. Climate change may not necessarily have been the major factor $[4,9]$. Thus, the virus was imported to a town in northern Italy by a traveller returning from India, while human activities had altered the local ecology allowing establishment of the mosquito vector, Aedes albopictus. The eggs of the mosquito may have been introduced in cargo containers containing shipments of loose tyres imported from Asia [9]. The ability of the virus itself to respond to change is an important factor. Thus, for example, a single mutation in CHIKV has been identified as promoting infection in the mosquito A. albopictus over the recognized vector, Aedes aegypti [10]. This mutation increases the potential for CHIKV to permanently extend its range into Europe and the Americas, where A. albopictus has established over the last 20 years [11].

The objective of this study was to prioritize five vector-borne viruses according to the risk of incursion into the EU and the risk of becoming endemic within the EU, both at the present time and after the impact of climate change assumed to have occurred in the 2080s. In addition, the impact of climate change was broken down into the various routes of introduction. A qualitative risk-assessment approach based on elicitation of expert opinion was used. The impetus of
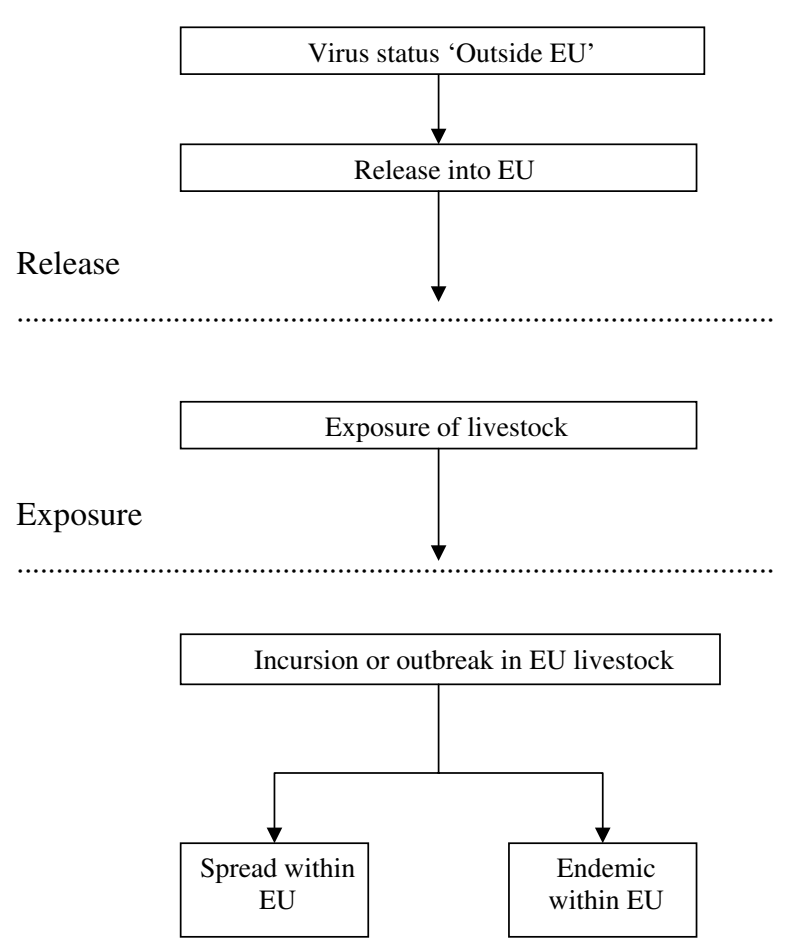

Consequence

Fig. 1. Risk pathway for the risk of vector borne livestock disease incursion, spread and becoming endemic in the EU.

this work was to facilitate the identification of appropriate risk-management options in relation to adaptations to climate change. The viruses studied were African horse sickness virus (AHSV), CCHFV, RVFV, African swine fever virus (ASFV) and WNV. AHSV is transmitted by Culicoides midges, ASFV and CCHFV by ticks, and RVFV and WNV by mosquitoes. The 2080s was selected as the decade to consider for the impact of climate change as previous studies [12-14] have generated predictions for the 2080 s period or 'by the end of the 21 st century'.

\section{METHODS}

A qualitative risk assessment for five vector-borne viruses listed as notifiable by the World Organisation for Animal Health [15], was conducted based on the Office International des Epizooties (OIE) framework [16] and therefore included an assessment of the probabilities of release, exposure and consequence. The risk pathway is presented in Figure 1 and the full definitions of the release, exposure and consequence assessments are set out by OIE [16]. In this context, release corresponds to the likelihood of entry of the 
virus into the EU, exposure considers the likelihood of susceptible animals becoming exposed to the virus, and consequence defines the likelihood of biological, environmental or economic consequences, and their likely magnitude. Exposure and consequence are conditional on release and exposure, respectively.

\section{Elicitation of data through expert opinion methodology}

These data were qualitative estimates of the probabilities of release, exposure and consequence and were obtained by elicitation of expert opinion through questionnaires using the modified-Delphi technique [17]. The approach was similar to that used previously for estimating the risk of importation of foot-andmouth disease into Europe [18]. A workshop enabled the 'conditioning' of the experts prior to their completing the questionnaire for a second time and facilitated clarification of any interpretation issues with the questionnaire.

\section{Structure of the questionnaire and accompanying information}

Each virus-specific questionnaire was divided into five parts. Part 1 asked for background information on the expert. Throughout the remainder of the questionnaire, the expert was asked to answer questions relating to the virus both currently, and predicted in the 2080s after climate change. In the 'top level' analysis, the experts were asked to assess the risk through all routes combined, while further questions within each part of the questionnaire addressed the probabilities of release, exposure and outbreak through each of six routes, namely infected vector, infected livestock, meat or meat products from infected animals, infected wild animals, infected pets and infected persons. Part 2 asked the experts to indicate the current and future (2080) risk of incursions/ outbreaks of the virus in parts of the world outside the EU to provide an indication of the worldwide prevalence. Part 3 elicited expert opinion on the effect of climate change on the risk of release into the EU, given the current and predicted worldwide prevalence in Part 2. In Part 4, the experts were asked to assess the effect of climate change on the risk of exposure to livestock within the EU, given that the virus has entered the EU through any of the routes in Part 3. The experts were asked to consider the effect that climate change may have on farming practices with respect to changes in intensity of farming and movement of animals, when making their judgement. In Part 5, expert opinion was elicited on the effect of climate change on the consequences. Three consequences were considered, namely risk of incursion (or outbreak) given exposure, risk of spread within the EU given incursion, and risk of becoming endemic in the EU given incursion (Fig. 1). Here, only the results for the risk of incursion and risk of becoming endemic are provided. Each expert completed the same questionnaire twice; the first prior to the workshop and the second during the second half of the workshop. Included with the first questionnaire was supportive literature on definitions and climate change scenarios. The climate change scenarios across Europe in the 2080s were described broadly in the documentation as higher temperatures particularly in southern Europe, increasing frequency of hot summers, a wetter northern Europe, a drier southern Europe and more extreme weather conditions with increased risks of heat wave, drought and flooding [12, 14, 19].

\section{The expert opinion workshop}

The results of the first round of questionnaires were presented to the experts at the workshop. A presentation on climate change in Europe was given to standardize the experts' understanding of the climate predictions for the 2080s. In addition, breakout groups promoted discussion on each of the following topics: (1) the impact of climate change on farming; (2) the vertebrate reservoir hosts in Europe; and (3) the impact of climate change on the pathogen/vector interaction. At the end of the workshop, each expert repeated the questionnaire for each of the five viruses without reference to their answers from the first questionnaire. Only data from the second questionnaire were used in the risk assessment.

\section{Selection and weighting of experts}

Experts were chosen from across Europe on the basis of their expertise in nine vector-borne viruses listed by the World Organization for Animal Health [15], namely AHSV, RVFV, CCHFV, ASFV, WNV, Venezuelan equine encephalitis virus, Western equine encephalitis virus, Japanese encephalitis virus and vesicular stomatitis virus. For the purpose of the workshop, five of those viruses were selected, namely AHSV, RVFV, CCHFV, ASFV and WNV, on the basis of expertise of the experts given in the first round 
Table 1. Summary of the expertise of the experts for each virus

\begin{tabular}{llrlll}
\hline \hline & \multicolumn{5}{l}{ Self assessment level of expertise } \\
\cline { 2 - 6 } Virus & Very low & Low & Medium & High & Very high \\
\hline AHSV & 2 & 9 & 5 & 0 & 1 \\
ASFV & 3 & 11 & 2 & 1 & 0 \\
CCHFV & 5 & 10 & 2 & 0 & 0 \\
RVFV & 3 & 12 & 2 & 0 & 0 \\
WNV & 0 & 8 & 5 & 3 & 1 \\
\hline \hline
\end{tabular}

AHSV, African horse sickness virus; ASFV, African swine fever virus; CCHFV, Crimean Congo haemorrhagic fever virus; RVFV, Rift Valley fever virus, WNV, West Nile virus.

Very high, Published many papers, led research projects on this virus, including an investigation of the impact of climate change.

High, Published many papers, led research projects on this virus.

Medium, Worked on research projects and contributed to papers on the virus.

Low, Some background knowledge, but no direct research experience on virus.

Very low, Minimal background knowledge of virus.

of questionnaires. In total, 18 experts were recruited. Of those, 16 completed the questionnaire individually, with two completing the questionnaire jointly. In total therefore, 17 questionnaires were completed for each virus. This was to enable a comparison at the group level and was judged to be an essential part of the process. It was also felt that although some experts may have considered their expertise to be very low for the virus per se, they may have information on the vector or the ecology, for example, or other factors which could be drawn upon. The questionnaire included a self-ranking of the expert's level of expertise for each virus (Table 1). To accommodate the differences between experts in their levels of expertise for each virus, each level of expertise was given a weighting $\left(W_{i, j}\right.$, where $i=$ number of expert, $i=1$, $\ldots, 17, j=$ virus). For each virus, a scoring system was used that gave a score of 5 for an expert with 'very high' expertise decreasing to a score of 1 for an expert with a 'very low' level of expertise. Consequently for each virus, an overall risk was obtained for each expert and was combined with the risks from the other experts by allowing each expert to contribute $W_{i, j}$ risks. For example, if expert 3 has expertise level 'medium' for WNV and gave an overall assessment of 'low' then that expert will contribute $3\left(W_{3, \mathrm{WNV}}=3\right)$ 'lows' to the overall assessment. Similarly, expert 5, who has a 'low' expertise in WNV but estimated the risk to be 'negligible' will contribute two 'negligibles' $\left(W_{5}\right.$,WNV $\left.=2\right)$. This method was applied across the experts to give a weighted distribution of the overall risk, from which a median value and 10th and 90th percentiles were derived. To investigate the impact of the weighting scheme on the results a sensitivity analysis was undertaken.

\section{Qualitative assessment of risk}

The definitions of the probabilities of an event occurring [20] were given to the experts in an accompanying information sheet as: negligible (so rare it does not merit consideration); low (rare but does occur); medium (occurs regularly); and high (occurs very regularly). Experts were asked to qualify the probabilities of release, exposure and consequence, given these definitions.

Due to the conditional nature of the exposure and consequence probabilities, the overall estimate of risk for a pathway (Fig. 1) can be derived by 'multiplying' the probabilities of release, exposure and consequence. A matrix was defined to determine the result of multiplying two qualitative probabilities (Table 2). The structure of the matrix accounts for the fact that probabilities are always between 0 and 1 . Therefore, when 'multiplying' probabilities together the resulting probability must be, at the absolute maximum, equal to the lower probability. Other matrix approaches [20] were also considered. However, these were not deemed appropriate because they do not capture the multiplicative nature of the risks being assessed here. For each virus and for each expert, the risk associated with each consequence was calculated for the current time and with climate change as predicted in 2080.

\section{Assessment and comparison of the risks through different routes of transmission}

The questionnaire specified six routes of entry of virus, namely vectors, livestock, wildlife, meat and meat products, persons and pets. While transmission of ASFV occurs from direct pig-to-pig contact, and also through consumption of meat products from infected pigs, transmission to livestock for the other four viruses studied here is primarily dependent on a competent vector. Thus, in the absence of a mosquito vector, the risk of direct transmission of $\mathrm{WNV}$, for example, from birds to horses would be negligible. It was therefore explained in the questionnaire that 
Table 2. Matrix used for multiplication of two qualitative probabilities

\begin{tabular}{lllll}
\hline \hline \multirow{2}{*}{$\begin{array}{l}\text { Results of } \\
\text { probability } 2\end{array}$} & Results of probability 1 & \\
\cline { 2 - 5 } & Negligible & Low & Medium & High \\
\hline Negligible & Negligible & Negligible & Negligible & Negligible \\
Low & Negligible & Low & Low & Low \\
Medium & Negligible & Low & Medium & Medium \\
High & Negligible & Low & Medium & High \\
\hline \hline
\end{tabular}

exposure through a given route encompasses both direct and indirect contact with the virus, given that the virus has entered the EU through that route. The questionnaire cited soil, air, water, food, direct contact with other animals and humans, contact with a vector or exposure through the mechanics of biting as examples.

\section{Sensitivity analysis}

The method used for the weighting of experts can be considered subjective. We therefore considered another two systems: no weighting of experts and nonlinear weighting. The weights associated with these systems are presented in Table 3 . Using the alternative weighting systems, the risk of incursion for AHSV, ASFV, CCHFV, RVFV and WNV in the EU were estimated and compared.

\section{RESULTS}

The risks for two consequences, namely the risk of incursion and the risk of the virus becoming endemic in the EU at the 'top level' (i.e. through all routes combined) are compared under the current situation and with climate change. In addition, the risk of incursion is broken down into the individual routes of entry. All results refer to risks, which were linearly weighted (Table 3) to accommodate the level of expertise of the experts for each virus. Table 1 summarizes the expertise for the five viruses.

\section{Risk of an incursion into the $\mathbf{E U}$ at the current time and with climate change}

At the current time, the predicted risk of incursion in the EU from other parts of the world was greater for ASFV and WNV than for AHSV, CCHFV, and RVFV (Table 4). Climate change increased the predicted median risk for AHSV from 'low' to 'medium' while the median risks for the other viruses were
Table 3. Alternative systems used in the sensitivity analysis for the weighting of experts

\begin{tabular}{llll}
\hline \hline & \multicolumn{2}{l}{ Weight $\left(W_{i, j}\right)$ assigned to expert $i$ for virus $j$} \\
\cline { 2 - 4 } $\begin{array}{l}\text { Definitions } \\
\text { of expertise } \\
\text { of experts }\end{array}$ & $\begin{array}{l}\text { No } \\
\text { weighting }\end{array}$ & $\begin{array}{l}\text { Linear } \\
\text { weighting } \\
\text { (baseline) }\end{array}$ & $\begin{array}{l}\text { Nonlinear } \\
\text { weighting }\end{array}$ \\
\hline Very high & 1 & 5 & 10 \\
High & 1 & 4 & 6 \\
Medium & 1 & 3 & 4 \\
Low & 1 & 2 & 2 \\
Very low & 1 & 1 & 1 \\
\hline \hline
\end{tabular}

unchanged (Table 4). This increase in risk for AHSV can be attributed to the experts' predicting an increase in the risk of both release and exposure (Fig. 1). For CCHFV and RVFV, climate change affected the 10th-90th percentiles, which is consistent with a small increase in predicted risk. This was due to an increase in the predicted risk of release and exposure for CCHFV and an increase in the risk of release (but not exposure) for RVFV with climate change (Fig. 1).

\section{Risk of virus becoming endemic in the $\mathrm{EU}$ at the current time and with climate change}

The current risk of becoming endemic was highest for $\mathrm{WNV}$, as indicated by the median and lowest for RVFV, as indicated by the 10th-90th percentiles (Table 5). Climate change was predicted to have no impact on the predicted median risk for any of the five viruses becoming endemic in the EU. However, a predicted impact was apparent when considering the 10th-90th percentiles. In particular, the 10th-90th percentiles shifted for RVFV, and the 10th percentiles for AHSV and CCHFV increased, suggesting that climate change may, in the opinion of some experts, increase the risk of these viruses becoming endemic in the EU. Climate change did not appear to affect the predicted 10th-90th percentile risks for either ASFV or WNV becoming endemic in the EU. Further 
Table 4. Qualitative assessment of the risk of incursion of five vector-borne livestock viruses in the EU

\begin{tabular}{|c|c|c|c|c|}
\hline \multirow[b]{2}{*}{ Virus } & \multicolumn{2}{|c|}{ Current risk } & \multicolumn{2}{|c|}{ Risk in 2080 s after climate change } \\
\hline & Median & 10th 90th percentile & Median & 10th 90 th percentile \\
\hline AHSV & Low & Low medium & Medium & Low medium \\
\hline ASFV & Medium & Low medium & Medium & Low medium \\
\hline $\mathrm{CCHFV}$ & Low & Negligible medium & Low & Low medium \\
\hline RVFV & Low & Negligible low & Low & Low medium \\
\hline WNV & Medium & Negligible medium & Medium & Negligible medium \\
\hline
\end{tabular}

AHSV, African horse sickness virus; ASFV, African swine fever virus; CCHFV, Crimean Congo haemorrhagic fever virus; RVFV, Rift Valley fever virus, WNV, West Nile virus.

Table 5. Qualitative assessment of the risk of five vector-borne livestock viruses becoming endemic in the EU

\begin{tabular}{|c|c|c|c|c|}
\hline \multirow[b]{2}{*}{ Virus } & \multicolumn{2}{|c|}{ Current risk } & \multicolumn{2}{|c|}{ Risk in 2080 s after climate change } \\
\hline & Median & 10th 90th percentile & Median & 10th 90th percentile \\
\hline AHSV & Low & Negligible medium & Low & Low medium \\
\hline ASFV & Low & Low medium & Low & Low medium \\
\hline $\mathrm{CCHFV}$ & Low & Negligible medium & Low & Low medium \\
\hline RVFV & Low & Negligible low & Low & Low medium \\
\hline WNV & Medium & Negligible medium & Medium & Negligible medium \\
\hline
\end{tabular}

AHSV, African horse sickness virus; ASFV, African swine fever virus; CCHFV, Crimean Congo haemorrhagic fever virus; RVFV, Rift Valley fever virus, WNV, West Nile virus.

investigation revealed that the AHSV percentiles increased due to predicted increases in the probabilities of release, exposure and becoming endemic given incursion, and that the percentiles for CCHFV increased due to increases in the probabilities of release, exposure and becoming endemic given incursion (Fig. 1). The RVFV percentiles increased due to the increase in the probabilities of release and becoming endemic given incursion.

The 'between-expert' variation, as described by the 10th-90th percentiles, for the risk of becoming endemic for AHSV, CCHFV and WNV indicated a considerable degree of disagreement between experts (negligible-medium). Interestingly, there seemed to be greater agreement between experts for the prediction with climate change in that the 10th-90th percentile ranges for AHSV and CCHFV narrowed to lowmedium. Agreement between experts was greater for RVFV and ASFV.

\section{Impact of climate change on the routes of incursion into the $\mathbf{E U}$}

The risks of incursion into the EU from entry of virus through each of the six routes were estimated for each virus. The results given in Table 6 show those routes of entry for which the predicted risk of incursion was greater than negligible. The current median risk of incursion from entry of vectors was predicted to be non-negligible for each of the five viruses, as expected for vector-borne viruses. However, only for AHSV and CCHFV were the current risks of incursion from entry of infected vectors predicted to be highest for the six routes of entry studied. Thus for ASFV, the risks of incursion currently and for the 2080s from entry of meat and meat products and livestock were predicted to be higher than that through the entry of vectors. For WNV, the risk of incursion through entry of wildlife was predicted to be the highest currently. This reflects mosquitoes' feeding on infected migratory birds. For RVFV, the risk of incursion through entry of livestock was predicted to be higher currently than that through entry of vectors as judged by the 10th-90th percentiles.

Climate change was predicted to increase the median risk of incursion through entry of vectors from low to medium for AHSV, CCHFV and WNV (Table 6). For these three viruses, the changes in risk of incursion through entry of vectors were due to the experts believing that a change in the release and 
Table 6. Qualitative assessment of the risk of incursion into the EU for five vector-borne virses: non-negligible routes of release

\begin{tabular}{llll}
\hline \hline \multirow{2}{*}{ Virus } & Risk from main routes, median (10th 90th percentile) & \\
\cline { 2 - 4 } AHSV & Main routes & Current risk & $\begin{array}{l}\text { Risk in 2080s after } \\
\text { climate change }\end{array}$ \\
& Vectors & Low (negligible medium) & Medium (low medium) \\
& Livestock & Low (negligible medium) & Low (negligible medium) \\
ASFV & Wildlife & Negligible (negligible medium) & Low (negligible medium) \\
& Livestock & Medium (low medium) & Medium (low medium) \\
& Vectors & Low (negligible medium) & Low (low medium) \\
CCHFV & Vectors & Low (negligible low) & Low (negligible medium) \\
& Livestock & Low (negligible medium) & Medium (low medium) \\
& Wildlife & Low (negligible medium) & Low (negligible medium) \\
RVFV & Livestock & Low (negligible low) & Low (negligible medium) \\
& Vectors & Low (negligible medium) & Low (low medium) \\
& Wildlife & Low (negligible low) & Low (low medium) \\
& Wildlife & Negligible (negligible low) & Low (negligible low) \\
WNV & Vectors & Medium (negligible medium) & Medium (negligible medium) \\
& Livestock & Low (low medium) & Medium (low medium) \\
& Negligible (negligible low) & Low (negligible low)
\end{tabular}

AHSV, African horse sickness virus; ASFV, African swine fever virus; CCHFV, Crimean Congo haemorrhagic fever virus; RVFV, Rift Valley fever virus, WNV, West Nile virus.

exposure probabilities would occur with climate change (Fig. 1), therefore suggesting that the vector range and/or vector density would increase. The predicted median risks of incursion through entry of vectors for ASFV and RVFV were not affected by climate change, but an increase was detected in the 90th percentile and 10th-90th percentile range, respectively, suggesting that the risk of these viruses through the vector route may also be increased by climate change.

It was predicted that climate change could increase the risks of incursion through entry of livestock for WNV, ASFV and RVFV to some degree. The predicted risk of ASFV through entry of meat and meat products from infected animals was not affected by climate change. Climate change was predicted to result in some increase in the risk of incursion through entry of wildlife for AHSV, RVFV and CCHFV, but not for WNV. This may reflect vectors' feeding on infected wildlife, either imported or entering the EU through migration.

\section{Sensitivity analysis: risk of incursion}

Re-analysing the expert opinion data using the additional expert weightings given in Table 3 (no weighting and nonlinear weighting) identified that the analysis was not very sensitive to the weights used. Comparing the risk of incursion (current climate) the only difference in the predicted median risks was for WNV. Thus the median was estimated to be medium for the no weighting and linear weighting, but was estimated to be low if using the nonlinear weighting. In addition, the 10th-90th percentile for ASFV was affected, being low-medium for no weighting and linear weighting, but negligible-medium for the nonlinear weighting. Under the 2080s climate change scenario, the only difference was for CCHFV with a medium estimate for the median risk being estimated when assuming the experts are equal in their expertise, but otherwise (linear and nonlinear weighting) the median was predicted to be low.

\section{DISCUSSION}

Investigations of climate changes during the last century suggest that the climate of Europe is changing at a rapid rate [21] and this development will most likely continue during the next decades [22]. These climatic changes are particularly pronounced in central Europe. Climate change may impact on livestock diseases through its effect on a number of factors including the range and abundance of vectors and wildlife reservoirs, survival of pathogens 
in the environment, and farming practice [8]. These factors may interact with each other and also with social and anthropogenic changes, including habitat destruction and changes in land use, which occur both globally and locally, and increased mobility of people and movement of goods including livestock [9].

In the case of BTV, incursion into southern Europe was supported by an expansion in range of its midge vector, $C$. imicola, from North Africa across the Mediterranean Sea through climate change [7]. Furthermore, higher temperatures increase the competence of the midge vector to transmit the virus between livestock [7, 23]. The importance of climate change in emergence of tick-borne viruses is less clear [24], and other factors such as social, political and economic changes have been shown to be important for tick-borne encephalitis virus (TBEV) in humans in the Baltic states [25]. In several countries in Europe, the dramatic spike in TBEV cases in humans in 2006 may have reflected favourable weather conditions promoting outdoor recreational activities, rather than changes in tick abundance [26]. For mosquito-borne viruses, there is also evidence from the WNV outbreak in Israel in 2000 that the magnitude of the minimum temperature during prolonged heat waves is the key climatic variable [27]. The 1999 outbreak in New York was preceded by a 3-month drought and a 2-week heat wave [28].

The qualitative risk assessment developed here focused on the impact of climate change on the release, exposure and consequence for five vector-borne viruses, and was parameterized by elicitation of expert opinion. Issues such as the impact of climate change on the vector, host reservoir or characteristics and epidemiology of the pathogen, although implicit in the expert responses, were not specifically addressed. The information given to the experts on the predictions for climate change in Europe was broad and, furthermore, it was not possible to regionalize Europe in terms of climatic predictions for the 2080s. Experts were therefore asked to consider Europe as a whole, although it is well-known that regional differences in climate within Europe are important for tick-borne diseases. For example, TBEV foci in central Europe reflect areas where the climatic conditions allow the temporal synchrony required for tick-to-tick transmission, and predictions for the 2080s suggest that TBEV will be eliminated from central Europe due to breaking of the synchrony with the last foci remaining in Scandinavia in the 2080s [29].
The expertise of the experts differed for the five viruses with expertise across the group being greater for WNV and AHSV and lowest for CCHFV. However, it should be noted that the definition of 'low' expertise (Table 1) includes some background knowledge, albeit no direct research experience on the virus. In the case of CCHFV therefore, 12 of the 17 experts had some background knowledge or greater. The work presented here demonstrates the approach and preliminary results from a group of experts initially selected to cover nine vector-borne livestock viruses. For the purpose of the workshop, it was felt that five was the maximum number of viruses which could be covered, and these were selected based on the expertise of the experts. Future approaches could focus on selecting experts for just those five viruses. The sensitivity analysis showed that weighting of expertise had relatively little effect on the conclusions. This is probably due to the matrix used (Table 2). The overall risks of incursion into the EU of AHSV, RVFV and CCHFV from outside the EU were predicted to be increased by climate change while the risks for WNV and ASFV were predicted not to be affected (Table 4). Similar trends were predicted for the risks of becoming endemic in the EU (Table 5). The predicted risk of incursion through the entry of vectors was increased by climate change for all five viruses, the effect being strongest for AHSV, CCHFV and WNV according to the medians (Table 6).

In the case of AHSV, this prediction is not surprising and the effect of climate change on the risk from Culicoides-transmitted viruses to the UK's livestock industry has been known for some time [23]. First, incursions of AHSV beyond its endemic areas (sub-Saharan Africa) have already occurred into the EU with outbreaks in Spain and Portugal [30]. Second, the most important vector for AHSV, the biting midge $C$. imicola, has recently expanded its range northwards from northern Africa into southern Europe to include not only Portugal and Spain, but also Italy, Greece and even southern Switzerland. This is believed to be due to climate change [7]. Third, as with the related BTV, higher temperatures increase vector competence, such that novel midge species, including those abundant in northern Europe (e.g. the C. obsoletus complex), may be able to serve as vectors enabling AHSV to extend its range well beyond that of C. imicola [5, 31]. The distribution and spatial coincidence of $C$. imicola and the $C$. obsoletus group throughout the Iberian Peninsula has recently been modelled [31]. 
Epidemics of CCHFV were first recorded in the Balkans in 1944 and in Africa in 1956 with outbreaks in Mauritania in 2003 [32] and Turkey in 2001-2008 [33]. Although the virus has been isolated from several genera and species of Ixodid ticks, the main vectors involved in CCHFV transmission are ticks of the genus Hyalomma, particularly Hyalomma marginatum. For CCHFV, climate change is predicted to increase the risks of incursion through entry of both vectors and wildlife (Table 6). The wildlife reservoirs for CCHFV are small mammals, including hares, hedgehogs and rats [34], which may benefit and increase in abundance through milder winters and enhanced heavy rainfall [35]. It has been argued that migratory birds are not the reason for the sudden appearance of CCHFV in Turkey [33]. Climate change and environmental changes may affect CCHFV epidemiology and trigger community outbreaks [1]. However, long-term trends in changing climate do not provide a sufficiently consistent explanation for the emergence of CCHFV in Turkey [33]. Thus, it has been proposed that changes in human behaviour directly affecting the density of vertebrate host reservoirs are more important than climatic factors [33]. In particular, war and terrorism in the region disrupted normal agricultural and hunting activities allowing weeds to grow and hare densities to increase. Although terrorist activities and war may have great impacts on the current and past emergences of CCHFV in Turkey and Crimea, climate change should not be neglected because certain environmental variables may affect the future dispersion and distribution of the Hyalomma vectors as well as that of the vertebrate reservoirs and livestock within Europe, and, furthermore, may affect the release of the virus from other parts of the world. It is possible that climate change will facilitate an expansion in the range of H. marginatum in Europe with creation of drier habitats. Both climate suitability for the Hyalomma tick vector and landscape fragmentation (through interspersion of agricultural land with shrub-type vegetation and forest) have been identified as important predictors of CCHFV cases in humans in Turkey [36]. Fragmentation of the landscape and habitats may be affected by climate change in the future with decreasing rainfall increasing the grassland between forested areas.

The prediction that climate change has little impact on the overall risk of incursion of ASFV is consistent with the prediction of climate change having no effect on the risk through the main route which was identified as meat and meat products (Table 6). Climate change does increase the predicted risk of incursion of ASFV through the entry of vectors (increase in the 90th percentile), but since this is a minor route compared to entry of meat and meat products, it has little impact on the overall risk for ASFV (Table 4). Ornithodoros (soft) ticks are the vectors for ASFV with Ornithodoros moubata, O. erraticus and O. sonrai serving as natural vectors in southern Africa, the Iberian Peninsula and recently Senegal, respectively [37]. It should be noted that climate change has brought about the expansion in range of Ornithodoros ticks species. Of particular interest here is that the soft tick, Ornithodoros (formerly Alectorobius) sonrai, has expanded its range south of the Sahara in response to drought, moving with the $750 \mathrm{~mm}$ isohyet [38]. Although it could be argued that warmer, drier climates could enable the expansion in range of soft ticks of the genus Ornithodoros within the EU, this would affect the risk of ASFV becoming endemic. Indeed, ASFV-infected ticks may provide a reservoir of the disease, for example through transovarial transmission [39], making its elimination more problematic, similar to Portugal [40].

The predicted current risks of incursion (Table 4) and becoming endemic (Table 5) are highest for $\mathrm{WNV}$, reflecting the fact that the virus is circulating in some EU countries. WNV is transmitted by mosquito vectors with birds being the host reservoir. Currently, the predicted risks of incursion for WNV through entry of wildlife (Table 6) are greater than that predicted from entry of vectors, livestock, pets and humans. This risk through wildlife may reflect mosquitoes' feeding on infected migratory birds. Indeed, the wide range and large numbers of susceptible species of migratory birds have been important in the dispersal of WNV throughout the Americas [4]. Elicitation of expert opinion concluded that the risk of incursion through entry of wildlife is unaffected by climate change (Table 6) suggesting that wild bird movements will be relatively unaffected by climate change. Indeed, climate change may cause a decline in the abundance of some long-distance migratory birds [41]. However, of great interest is the prediction that climate change will increase the risk of WNV incursion through entry of vectors (Table 6). It was reported recently that evidence does not exist to support WNV activity in British birds of different species tested, including passerines and corvids [42], which contradicts previous studies demonstrating WNV serological activity in the UK [43]. Over the next two decades, the UK may become more permissive to 
WNV survival and spread [30]. Factors include increasing mosquito numbers, newly arrived competent mosquito species, newly arrived virulent strains of the disease, and a greater tendency for UK citizens to spend the early evenings outdoors, when they may be bitten in the same sort of situations that appear to be important in the Americas. Simulations of climate change on Usutu virus (which is related to WNV) predict the frequency of outbreaks in blackbirds in the Vienna area of Austria increasing through the 21st century with the virus becoming endemic after 2040 [44]. It should be noted that local anthropogenic changes may also have important impact on the mosquito vectors for WNV in Europe [45]. In the Camargue region of France, for instance, the abundance of Culex modestus, which is the main mosquito vector for WNV, has been affected by expansion of rice cultivation, pesticide use and pest-management strategies [45].

The lowest risk range was predicted for RVFV (Tables 4 and 5), which is consistent with RVFV being the only one of the five viruses studied here which has not occurred in the EU to date. Although the median risks are unaffected by climate change, the 10th-90th percentiles for risk of incursion (Table 4) increased from negligible-low to low-medium with climate change, mirroring the increase in risk of incursion of RVFV predicted from entry of vectors (Table 6). The main vectors for RVFV are mosquitoes. During periods of drought, the virus survives in eggs of certain species of Aedes mosquitoes that hatch when rainfall occurs [9]. It has been suggested that extreme weather events such as floods and droughts, may create the necessary conditions for RVFV to expand its geographical range northwards and across the Mediterranean [46]. RVFV is one of the major vectorborne zoonoses in Africa, and human and animal cases were detected in the Arabian Peninsula for the first time in 2000. A study of the environmental and animal risk factors associated with RVFV in southwest Saudi Arabia identified associations between the disease and a dense mosquito population, high rainfall and the presence of ponds [47]. For RVFV, the predicted risk of incursion through entry of livestock is currently greater than for entry of wildlife (Table 6). The completion of a trans-Sahara road will heighten the risk of introduction of RVFV north of the Sahara [9]. Major trade in animals already exists between Africa and the Arabian Peninsula with an estimated seven million animals exported to Saudi Arabia during the pilgrimage seasons every year. Movement of infected animals and mosquito vectors, in addition to climate change, will determine whether RVFV disperses beyond its current boundaries [4].

In an assessment of 45 infectious and parasitic diseases, four vector-borne arboviruses were classified as being of high importance in France [48]. That study [48] concluded that with climate change, the probability of the evolution of the epidemiological situation of disease in France was negligible to low for RVFV, low to moderate for AHSV and high for WNV (and BTV). It is difficult to directly compare results, since those of the French study [48] are relevant to France only and not to the whole EU as is considered here. In addition, the analysis here breaks the risks down into the risk of incursion and risk of becoming endemic.

Breaking down the risks into the specific release routes provides further information to assist risk managers. The risk predictions made here through elicitation of expert opinion should be used in conjunction with consideration of the impact of climate change on vertebrate host reservoirs, arthropod vectors, farming practice and land use, together with an understanding of the biology of virus-vector-host interaction. The two approaches together provide a powerful tool for furthering our understanding of the potential impact of climate change on the emergence of vector-borne viruses, currently exotic to the EU.

\section{ACKNOWLEDGEMENTS}

This work was funded under work package 7.4 by EPIZONE, a Network of Excellence for Epizootic Disease Diagnosis and Control. We acknowledge the contributions of Trevor Drew (Veterinary Laboratories Agency, UK) and Ann Lindberg (National Veterinary Institute, Sweden) during the development of the questionnaire. We would like to thank the 18 expert scientists who participated; Paul Phipps (Veterinary Laboratories Agency, UK), Philip Mellor, Linda Dixon, Anthony Wilson (Institute of Animal Health, UK), Norbert Nowotny (University of Veterinary Medicine, Vienna, Austria), Stuart Perkins (Defence Science and Technology Laboratory, UK), Sylvie Lecollinet (Laboratoire d'études et de recherches en pathologie animale et zoonoses, France), Arife Erturk (Etlik Central Veterinary Control and Research Institute, Turkey), Jeroen Kortekaas (Animal Sciences Group, Wageningen University Research, The Netherlands), Paul Reiter (Institut Pasteur, France), Kurt Pfister 
(Ludwig-Maximilians-Universitaet Muenchen, Germany), Alessandra della Torre (University of Roma La Sapienza, Italy), Gioia Capelli, Paola De Benedictis (Istituto Zooprofilattico Sperimentale delle Venezie, Italy), Ulla Carlsson, Lena Renstrom (National Veterinary Institute, Sweden), Michele Dottori (Istituto Zooprofilattico of Lombardia and the Emilia Romagna, Italy) and Federica Monaco (Istituto Zooprofilattico Sperimentale dell'Abruzzo e del Molise G Caporale, Italy). We thank John Gloster of the Met Office (UK) for his presentation on climate change in Europe at the workshop.

\section{DECLARATION OF INTEREST}

None.

\section{REFERENCES}

1. Vorou R, Pierroutsakos IN, Maltezou HC. Crimean Congo haemorrhagic fever. Current Opinion in In fectious Diseases 2007; 20: 495500.

2. Fakoorziba MR, et al. Tick borne Crimean Congo haemorrhagic fever in Fars Province, southern Iran: epidemiologic characteristics and vector surveillance. Pakistan Journal of Biological Sciences 2006; 9: 26812684.

3. Baylis N, Githeko AK. The effects of climate change on infectious diseases of animals. T7.3. Foresight. In fectious diseases: preparing for the future. Office of Science and Innovation (http://www.foresight.gov.uk/ Infectious\%20Diseases/t7_3.pdf). Accessed 16 Febru ary 2009.

4. Gould EA, Higgs S. Impact of climate change and other factors emerging arbovirus diseases. Transactions of the Royal Society of Tropical Medicine and Hygiene 2009; 103: 109121.

5. Mehlhorn H, et al. First occurrence of Culicoides obsoletus transmitted Bluetongue virus epidemic in Central Europe. Parasitology Research 2007; 101: 14321955.

6. Landeg F. Bluetongue outbreak in the UK. Veterinary Record 2007; 161: 534.

7. Purse BV, et al. Climate change and the recent emerg ence of bluetongue in Europe. Nature Reviews Micro biology 2005; 3: 171181 .

8. Gale $\mathbf{P}$, et $\boldsymbol{a l}$. The effect of climate change on the oc currence and prevalence of livestock diseases in Great Britain: a review. Journal of Applied Microbiology 2009; 106: 14091423.

9. Reiter P. Climate change and mosquito borne disease: knowing the horse before hitching the cart. Revue Scientifique et Technique Office International des Epi zooties 2008; 27: 383398.

10. Tsetsarkin K, et al. A single mutation in Chikungunya virus affects vector specificity and endemic potential PLOS Pathogens 2007; 3: 18951906.
11. Medlock JM, et al. Analysis of the potential for survival and seasonal activity of Aedes albopictus (Diptera: Culicidae) in the United Kingdom. Journal of Vector Ecology 2006; 31 : 292304.

12. IPCC. Climate change 2001: Synthesis report. Cambridge: Cambridge University Press, 2001, pp. 6078 (http://www.ipcc.ch). Accessed 16 February 2009.

13. Hulme M, et al. Climate Change Scenarios for the United Kingdom: The UKCIP02 Scientific Report. Norwich: Tyndall Centre for Climate Change Re search, School of Environmental Sciences, University of East Anglia, 2002 (http://www.ukcip.org.uk). Accessed 16 February 2009.

14. Christensen JH, Christensen OB. Severe summertime flooding in Europe. Nature 2003; 421: 805806.

15. Office International des Epizooties. OIE listed diseases. (http://www.oie.int/eng/maladies/en_classification.htm). Accessed 16 February 2009.

16. Office International des Epizooties. Applying the OIE risk analysis framework. In: Handbook on Import Risk Analysis for Animals and Animal Products. Volume 1, Introduction and Qualitative Risk Analysis. Paris: World Organization for Animal Health, 2004, pp. 3153.

17. Ayyub BM. Experts, opinions and elicitation methods. In: Elicitation of Expert Opinions for Uncertainty and Risks. London: CRC Press, 2001, pp. 97124.

18. Gallagher E, et al. Estimating the risk of importation of foot and mouth disease into Europe. Veterinary Record 2002; 150: 769772 .

19. Beniston M, Diaz HF. The 2003 heat wave as example of summers in a greenhouse climate? Observations and climate model simulations for Basel, Switzerland. Global and Planetary Change 2004; 44: 7381

20. Moutou F, Dufour B, Ivanov Y. A qualitative assessment of the risk of introducing foot and mouth disease into Russia and Europe from Georgia, Armenia and Azerbaijan. Revue Scientifique et Technique Office International des Epizooties 2001; 20: 723730.

21. Gerstengarbe FW, Werner PC. Climate development in the last century global and regional. International Journal of Medical Microbiology 2008; 298 (Suppl. 1): 511.

22. IPCC. IPCC 4th Assessment Report AR4, IPCC Secretariat, World Meteorological Organization, Switzerland, 2007 (http://www.ipcc.ch/pdf/assessment report/ar4/syr/ar4_syr.pdf). Accessed 27 April 2009.

23. Whitmann EJ, Baylis M. Climate change: effects on Culicoides transmitted viruses and implications for the UK. Veterinary Journal 2000; 160: 107117.

24. Randolph SE. Dynamics of tick borne disease systems: minor role of recent climate change. Revue Scientifique et Technique Office International des Epizooties 2008; 27: 367381 .

25. Sumilo D, et al. Tick borne encephalitis in the Baltic States: Identifying risk factors in space and time. International Journal of Medical Microbiology 2006; 296 (Suppl. 1): 7679

26. Randolph S, et al. Variable spikes in tick borne encephalitis incidence in 2006 independent of variable 
tick abundance but related to weather. Parasites and Vectors 2008; 1 : 44.

27. Paz S. The West Nile virus outbreak in Israel (2000) from a new perspective: the regional impact of climate. International Journal of Environmental Health Research 2006; 16: 113.

28. Rogers DJ, Sanderson V. West Nile virus: drivers, sources and receptors. Foresight T5.12 Infectious dis eases: preparing for the future. Office of Science and Innovation (http://www.foresight.gov.uk/Infectious \% 20Diseases/t5_12.pdf). Accessed 16 Feb 2009.

29. Rogers DJ, Randolph SE. Climate change and vector borne diseases. Advances in Parasitology 2006; 62: 345 381.

30. Mellor PS, Hamblin C. African horse sickness. Veterinary Research 2004; 35: 445466.

31. Calvete $\mathbf{C}$, et al. Modelling the distributions and spatial coincidence of bluetongue vectors Culicoides imicola and the Culicoides obsoletus group through the Iberian pen insula. Medical Veterinary Entomology 2008; 22: 124134.

32. Nabeth P, et al. Crimean Congo Hemorrhagic Fever, Mauritania. Emerging Infectious Diseases 2004; 10: 21432149.

33. Randolph S, Ergonul O. Crimean Congo haemorrhagic fever: exceptional epidemic of viral haemorrhagic fever in Turkey. Future Virology 2008; 3: 303306.

34. Nalca A, Whitehouse CA. Crimean Congo hemorrhagic fever virus infection among animals. In: Ergonil O, Whitehouse CA, eds. Crimean Congo Hemorrhagic Fever A Global Perspective. Dordrecht, Netherlands: Springer, 2007, pp. 155166.

35. Carrara AS, et al. Venezuelan equine encephalitis virus infection of cotton rats. Emerging Infectious Diseases 2007; 13: 11581165.

36. Estrada-Peña A, et al. Modeling the spatial distribution of Crimean Congo hemorrhagic fever outbreaks in Turkey. Vector Borne Zoonotic Diseases 2007; 7: 667 678.

37. Vial L, et al. African swine fever virus DNA in soft ticks, Senegal. Emerging Infectious Diseases 2007; 13: 19281931.

38. Trape JF, et al. The spread of tick borne borreliosis in West Africa and its relationship to sub Saharan drought. American Journal of Tropical Medicine and Hygiene 1996; 54: 289293.

39. Rennie L, Wilkinson PJ, Mellor PS. Transovarial transmission of African swine fever virus in the argasid tick Ornithodoros moubata. Medical and Veterinary Entomology 2001; 15: 140146.

40. APHIS (Animal and Plant Health Inspection Service Veterinary Services). Evaluation of the African swine fever status of Portugal. (https://web01.aphis.usda.gov/ db/mtaddr.nsf/0/2e8a32fada37ccce85256b12004d8c64/ \$FILE/portugal \%20ASF \%20reportr.pdf). Accessed 16 February 2009.

41. Both C, et al. Climate change and population declines in a long distance migratory bird. Nature 2006; 441 : 8183.

42. Phipps LP, et al. Surveillance for West Nile virus in British birds (2001 to 2006). Veterinary Record 2008; 162: 413415.

43. Buckley A, et al. Serological evidence of West Nile virus, Usutu virus and Sindbis virus infection of birds in the UK. Journal of General Virology 2003; 84: 2807 2817.

44. Brugger K, Rubel F. Simulation of climate change sce narios to explain Usutu virus dynamics in Austria. Preventive Veterinary Medicine 2009; 88: 2431.

45. Poncon N, et al. Effects of local anthropogenic changes on potential malaria vector Anopheles hyrcanus and West Nile virus vector Culex modestus, Camargue, France. Emerging Infectious Diseases 2007; 13: 18101815.

46. Martin V, et al. The impact of climate change on the epidemiology and control of Rift Valley fever. Revue Scientifique et Technique Office International des Epi zooties 2008; 27: 413426.

47. Elfadil AA, Hasab-Allah KA, Dafa-Allah OM. Factors associated with Rift Valley fever in south west Saudi Arabia. Revue Scientifique et Technique Office Inter national des Epizooties 2006; 25 : 11371145.

48. Dufour B, et al. A method to rank the risks of infectious diseases development linked to global warming. Pro ceedings of the 11th International Symposium on Veter inary Epidemiology and Economics (www.sciquest.org. nz). Cairns, Australia, 611 August 2006. Accessed 2 November 2007. 